\title{
$\sigma$-POROSITY IN MONOTONIC ANALYSIS WITH APPLICATIONS TO OPTIMIZATION
}

\author{
A. M. RUBINOV \\ Received 14 November 2003
}

We introduce and study some metric spaces of increasing positively homogeneous (IPH) functions, decreasing functions, and conormal (upward) sets. We prove that the complements of the subset of strictly increasing IPH functions, of the subset of strictly decreasing functions, and of the subset of strictly conormal sets are $\sigma$-porous in corresponding spaces. Some applications to optimization are given.

\section{Introduction}

Consider a normed space $X$ equipped with the order relation $\geq$ induced by a convex open cone $C$ such that $\mathrm{cl} C$ is a pointed cone. (More general conic sets $C$ are considered in some parts of the paper.) We study metric spaces of increasing positively homogeneous of degree one (IPH) functions defined on $C$, decreasing nonnegative functions defined on $C$, and conormal (upward) subsets of $C$. Recall that the set $V \subset C$ is called conormal if $(x \in V, y \geq x) \Rightarrow y \in V$. We show that the complements of the subset of strictly increasing IPH functions, of the subset of strictly decreasing functions, and of the subset of strictly conormal sets are $\sigma$-porous in the corresponding spaces. First results of this kind were presented in [10]. We use a modification of some constructions from [10] in this paper.

There are some links between IPH functions, decreasing functions, and conormal sets. These links are based on the following observation: consider a set $V \subset C_{*}:=C \times(0,+\infty)$. Then the following assertions are equivalent.

(1) $V$ is a closed and conormal set.

(2) $V$ is the upper-level set $\{x: p(x) \geq 1\}$ of an IPH function $p$ defined on $C_{*}$.

(3) $V$ is the epigraph of a lower semicontinuous nonnegative decreasing function $g$ defined on $C$.

Using corresponding bijections we can extend results obtained for metric spaces of IPH functions to metric spaces of conormal sets and decreasing functions.

The main results are obtained for the set $\mathscr{P}_{0}$ of IPH functions $p$ such that $0<$ $\inf _{x \in C,\|x\|=1} p(x) \leq \sup _{x \in C,\|x\|=1} p(x)<+\infty$. The number

$$
\rho(p, q)=\max \left(\sup _{x \in C} \frac{p(x)}{q(x)}, \sup _{x \in C} \frac{q(x)}{p(x)}\right)
$$


can be considered as a natural measure for the estimation of the closeness of functions $p, q \in \mathscr{P}_{0}$. This observation leads to the introduction of the natural metric $d(p, q)=$ $\ln \rho(p, q)$ on the set $\mathscr{P}_{0}$. It is easy to see that the metric space $\left(\mathscr{P}_{0}, d\right)$ is complete. Note that $d(p, q)$ does not depend on the "size" of the pair $p, q$ in the sense that $d(p, q)=d(\lambda p, \lambda q)$ for all $\lambda>0$. This number describes the distance in terms of the "shape" of this pair. Using above-mentioned bijections we introduce metric spaces of conormal sets and decreasing nonnegative functions and study their properties.

We also discuss applications of the results obtained to the examination of some questions arising in optimization. Consider the set $H$ of optimization problems $P(f, g)$ :

$$
\text { minimize } f(x) \text { subject to } g(x) \leq 0 \text {, }
$$

where $f, g: \mathbb{R}^{m} \rightarrow \mathbb{R}$ are functions with some properties. Many questions related to problem $P(f, g)$ can be expressed in terms of the perturbation function $\beta_{f, g}(y)=\inf _{x: g(x) \leq y} f(x)$. This function is decreasing. We say that problems $P(f, g) \in H$ and $P\left(f^{\prime}, g^{\prime}\right) \in H$ are equivalent if $\beta_{f, g}=\beta_{f^{\prime}, g^{\prime}}$. Thus we can include the set $\mathscr{H}$ of classes of equivalent pairs of optimization problems in the metric space of decreasing functions. Using results obtained, we can prove that the subset of $\mathscr{H}$ that consists of pairs $\pi$ such that at least one problem from $\pi$ has no solutions on the boundary of the set of feasible elements, is $\sigma$ porous in the metric space under consideration.

\section{Preliminaries}

A set $C$ in a normed space $X$ is called conic if $\lambda x \in C$ for all $x \in C$ and $\lambda>0$. A convex conic set is called a convex cone. A convex cone $K$ generates the order relation $\geq$ on $X$, namely $x \geq y$ if $x-y \in K$. We will write $x>y$ if $x \geq y$ and $x \neq y$. Let $C$ be a conic set. A function $p: C \rightarrow \mathbb{R}_{+\infty}$ is called positively homogeneous of degree one (PH) if $p(\lambda x)=$ $\lambda p(x)$ for all $\lambda>0$. Denote by $\mathscr{B}$ the intersection of $C$ and the unit ball and by $\mathscr{S}$ the intersection of $C$ and the unit sphere:

$$
\mathscr{B}=\{x \in C:\|x\| \leq 1\}, \quad \mathscr{S}=\{x \in C:\|x\|=1\} .
$$

Clearly each IPH function is completely defined by its trace on $\mathscr{S}$ : if $p_{1}, p_{2}$ are PH functions and $p_{1}(x)=p_{2}(x)$ for all $x \in \mathscr{Y}$, then $p_{1}(x)=p_{2}(x)$ for all $x \in C$. Let $p: C \rightarrow \mathbb{R}$ be a finite $\mathrm{PH}$ function. The quantity

$$
\|p\|=\sup \{|p(x)|: x \in \mathscr{B}\}=\sup \{|p(x)|: x \in \mathscr{G}\}
$$

is called the norm of $p$. The following simple assertion is well known and can be easily proved.

Proposition 2.1. A finite PH function $p$ defined on a conic set $C$ is continuous at zero if and only if $\|p\|<+\infty$.

Let $C$ be a conic set in a normed space $X$ with the order relation $\geq$ induced by a closed convex pointed cone $K \supset C$. (Recall that $K$ is called pointed if either $K \cap(-K)=\varnothing$ or $K \cap(-K)=\{0\}$.) A function $p: C \rightarrow \mathbb{R}$ is called increasing if $x \geq y \Rightarrow p(x) \geq p(y)$. 
A function $p: C \rightarrow \mathbb{R}$ is called strictly increasing if $x>y \Rightarrow p(x)>p(y)$. In this paper, we will study increasing and strictly increasing $\mathrm{PH}$ functions. We will use the abbreviation IPH for increasing PH functions. Since the ordering convex cone $K$ contains $C$, it follows that $x \geq 0$ for each $x \in C$.

The following proposition holds.

Proposition 2.2. Let $C$ be a conic set with int $C \neq \varnothing$ and let the order relation $\geq$ be induced by a closed convex cone $K \supset C$. Let $p: C \rightarrow \mathbb{R}_{+\infty}$ be an IPH function. Then

(i) $p$ is nonnegative;

(ii) if there exists $x \in C$ such that $p(x)=+\infty$, then $p(y)=+\infty$ for all $y \in \operatorname{int} C$;

(iii) if there exists $x \in C$ such that $p(x)>0$, then $p(y)>0$ for all $y \in \operatorname{int} C$.

A proof of Proposition 2.2 for the case $C=K$ can be found in [3, Proposition 6]. This proof is valid in the general case and we omit it.

We denote by $\mathscr{P}_{*}$ the set of all nonnegative $\mathrm{PH}$ functions defined on $C$ and continuous at zero. We assume that $\mathscr{P}_{*}$ is equipped with a metric $d_{u}$ defined by

$$
d_{u}(p, q)=\|p-q\|:=\sup \{|p(x)-q(x)|: x \in \mathscr{Y}\} .
$$

Clearly $d_{u}(p, q)=\sup \{|p(x)-q(x)|: x \in \mathscr{B}\}$. Clearly the metric space $\left(\mathscr{P}_{*}, d_{u}\right)$ is complete.

\section{Metric spaces of equivalent IPH functions}

Let $C$ be a conic set in a normed space $X$. Consider a conic set $\mathscr{P} \subset \mathscr{P}_{*}$ of continuous at zero nonnegative $\mathrm{PH}$ functions $p: C \rightarrow \mathbb{R}$. We assume that $\mathscr{P}$ is closed with respect to uniform convergence on $\mathscr{S}=\{x \in C:\|x\|=1\}$. We give some examples of a set $\mathscr{P}$ : the set of nonnegative and continuous at zero $\mathrm{PH}$ functions, the set of nonnegative continuous $\mathrm{PH}$ functions, the set of continuous at zero IPH functions (it is assumed that there is an order relation introduced by a closed convex pointed cone $K \supset C$ ). We say that functions $p, q \in \mathscr{P}$ are equivalent and write $p \sim q$ if there exist constants $0<\gamma \leq \Gamma<+\infty$ such that

$$
\gamma p(x) \leq q(x) \leq \Gamma p(x) \quad \forall x \in C .
$$

Clearly $\sim$ is an equivalency relation. Let $r \in \mathscr{P}$. Consider the class $\mathscr{P}^{r}$ of functions equivalent to a function $r$. It easily follows from the definition of the equivalence that $\mathscr{P}^{r}$ is a conic set. Assume in the sequel that $0 / 0=0$. Then the ratio $p(x) / q(x)$ is finite for all $x \in C$. If $p, q \in \mathscr{P}^{r}$, then

$$
\begin{aligned}
& \sup _{x \in C} \frac{p(x)}{q(x)}=\inf \{\gamma: p(x) \leq \gamma q(x) \forall x \in C\}<+\infty, \\
& \sup _{x \in C} \frac{q(x)}{p(x)}=\inf \{\gamma: q(x) \leq \gamma p(x) \forall x \in C\}<+\infty .
\end{aligned}
$$

Let

$$
\rho(p, q)=\max \left(\sup _{x \in C} \frac{p(x)}{q(x)} \sup _{x \in C} \frac{q(x)}{p(x)}\right), \quad p, q \in \mathscr{P}_{r} .
$$


It is easy to check that the function $\rho$ possesses the following properties:

$$
\begin{aligned}
\rho(p, q) & =\rho(q, p) \quad \forall p, q \in \mathscr{P}^{r} ; \\
\rho(p, q) & \geq 1 \quad \forall p, q \in \mathscr{P}^{r} ; \\
\rho(p, q) & =1 \quad \text { iff } p=q ; \\
\rho(p, h) & \leq \rho(p, q) \rho(q, h) \quad \forall p, q, h \in \mathscr{P}^{r} ; \\
\rho(\lambda p, \lambda q) & =\rho(p, q) \quad \forall p, q \in \mathscr{P}^{r}, \lambda>0 .
\end{aligned}
$$

We only check (3.7). Let $p, q \in \mathscr{P}^{r}$. Then we have for all $x \in C$

$$
\begin{aligned}
\frac{p(x)}{h(x)} & \leq \frac{p(x)}{q(x)} \frac{q(x)}{h(x)} \leq \rho(p, q) \rho(q, h) ; \\
\frac{h(x)}{p(x)} & \leq \frac{h(x)}{q(x)} \frac{q(x)}{p(x)} \leq \rho(q, h) \rho(p, q) .
\end{aligned}
$$

These inequalities imply (3.7). It follows from (3.4)-(3.7) that the function

$$
d(p, q)=\ln \rho(p, q), \quad p, q \in \mathscr{P}^{r}
$$

is a metric on $\mathscr{P}^{r}$. Note that $d$ is positively homogeneous of degree zero: $d(\lambda p, \lambda q)=$ $d(p, q)$ for all $p, q \in \mathscr{P}^{r}$ and $\lambda>0$. This follows from (3.8).

Proposition 3.1. Let $\alpha>0$ and let $\delta=e^{\alpha}-1$. Let $p, q \in \mathscr{P P}^{r}$. Then $d(p, q) \leq \alpha$ if and only if

$$
p(x)-q(x) \leq \delta q(x), \quad q(x)-p(x) \leq \delta p(x) \quad \forall x \in C .
$$

Proof. The definitions of $d$ and $\rho$ imply that

$$
d(p, q) \leq \alpha \Longleftrightarrow \rho(p, q) \leq e^{\alpha} \Longleftrightarrow\left(p(x) \leq e^{\alpha} q(x), q(x) \leq e^{\alpha} p(x) \forall x \in C\right) .
$$

Since $e^{\alpha}=1+\delta$, then (3.12) shows that $d(p, q) \leq \alpha$ if and only if (3.11) holds.

It follows from Proposition 3.1 that a sequence $p_{n}$ converges to $p$ in the metric space $\left(\mathscr{P}^{r}, d\right)$ if and only if there exists a sequence $\delta_{n}>0, \delta_{n} \rightarrow 0$ such that

$$
\begin{aligned}
& p_{n}(x)-p(x) \leq \delta_{n} p(x) \quad \forall x \in C, \\
& p(x)-p_{n}(x) \leq \delta_{n} p_{n}(x) \quad \forall x \in C .
\end{aligned}
$$

Proposition 3.2. Let $p_{n} \in \mathscr{P}^{r}, n=1, \ldots, p \in \mathscr{P}^{r}$. If the sequence $p_{n}$ converges to $p$ in the metric space $\left(\mathscr{P}^{r}, d\right)$, then $p_{n}$ converges to $p$ uniformly on the set $\mathscr{\mathscr { B }}=\{x \in C:\|x\| \leq 1\}$.

Proof. Let $d\left(p_{n}, p\right) \rightarrow 0$. Due to Proposition 3.1 there exists a sequence $\delta_{n} \rightarrow 0$ such that (3.13) and (3.14) hold. It follows from (3.13) that $p_{n}(x) \leq\left(1+\delta_{n}\right) p(x)$. Applying (3.14) we conclude that

$$
p(x)-p_{n}(x) \leq \delta_{n}\left(1+\delta_{n}\right) p(x) .
$$


Since $\sup \{p(x): x \in \mathscr{B}\}<+\infty$, it follows from (3.13) and (3.15) that $p_{n}(x) \rightarrow p(x)$ uniformly on $\mathscr{B}$.

Consider the set $\mathscr{P}_{0}$ of functions $p \in \mathscr{P}$ such that $\inf \{p(x): x \in \mathscr{T}\}>0$. Clearly each two functions $p, q \in \mathscr{P}_{0}$ are equivalent and $\mathscr{P}_{0}$ coincides with $\mathscr{P}^{r}$ for arbitrary $r \in \mathscr{P}_{0}$. The following assertion demonstrates that the convergence in the space $\left(\mathscr{P}_{0}, d\right)$ follows from uniform convergence, therefore (see Proposition 3.2) coincides with the uniform convergence.

Proposition 3.3. Let $p_{n} \in \mathscr{P}_{0}, n=1, \ldots$ and let $p \in \mathscr{P}_{0}$. If the sequence $p_{n}$ converges to $p$ uniformly on the set $\mathscr{Y}$, then $p_{n}$ converges to $p$ in the metric space $\left(\mathscr{P}_{0}, d\right)$.

Proof. Let $c=\inf \{p(x): x \in \mathscr{Y}\}$. Then $c>0$. Since $p_{n}$ converges to $p$ uniformly on $\mathscr{T}$, it follows that $c_{n}=\inf \left\{p_{n}(x): x \in \mathscr{S}\right\}>c / 2$ for large enough $n$. Let $\delta_{n} \rightarrow 0$ be a sequence such that $\left|p_{n}(x)-p(x)\right| \leq \delta_{n}$ for all $x \in \mathscr{Y}$. Then for all $x \in \mathscr{S}$ and large enough $n$ we have

$$
p_{n}(x)-p(x) \leq \frac{\delta_{n}}{c} p(x), \quad p(x)-p_{n}(x) \leq \frac{2 \delta_{n}}{c} p_{n}(x), \quad x \in \mathscr{Y} .
$$

Since $p$ and $p_{n}$ are positively homogeneous, it follows that (3.16) holds for all $x \in C$.

Proposition 3.4. The metric space $\left(\mathscr{P}_{0}, d\right)$ is complete.

Proof. Let $p_{n} \in \mathscr{P}_{0}$ be a sequence such that for each $\varepsilon>0$ there exists $N$ with the following property: for all $n>N$ and all natural $m$ we have $d\left(x_{n}, x_{n+m}\right)<\varepsilon$. It follows from Proposition 3.1 that for all $x \in \mathscr{S}$, we have $p_{n}(x)<(1+\delta) p_{n+m}(x)$ and $p_{n+m}(x)<(1+$ $\delta) p_{n}(x)$ with $\delta=e^{\varepsilon}-1$. These inequalities imply $\inf _{x \in \mathscr{Y}} p_{n+m}(x) \geq 1 /(1+\delta) \inf _{x \in \mathscr{Y}} p_{n}(x)$ and $\sup _{x \in \mathscr{S}} p_{n+m}(x)<(1+\delta) \sup _{x \in \mathscr{Y}} p_{n}(x), m=1, \ldots$ It is clear that for each $x \in C$ there exists $p(x)=\lim _{n} p_{n}(x)$ and $p \in \mathscr{P}_{0}$.

\section{Porosity results for metric spaces of IPH functions}

In this section, we consider a closed subspace $\mathscr{P}$ of the metric space $\left(\mathscr{P}_{*}, d_{u}\right)$ (see Section 2 for the definition of this space) that consists of IPH functions. In particular, it can be the set of continuous at zero IPH functions or continuous everywhere IPH functions. Since $\mathscr{P}$ is closed, it follows that the metric space $\left(\mathscr{P}, d_{u}\right)$ is complete. We will show that under some natural assumptions the complement of the set of strictly increasing $\mathrm{PH}$ functions from $\mathscr{P}$ is $\sigma$-porous in the space $\left(\mathscr{P}, d_{u}\right)$.

First we give the definition of porosity and $\sigma$-porosity, which will be used in the paper. There are various definitions of porocity (see [6] for references and a discussion). We use the following definition (see, e.g., $[1,2,5,10])$. Let $(P, d)$ be a complete metric space. Denote the ball $\{q \in P: d(p, q) \leq r\}$ by $B(p, r)$. A set $\Omega \subset X$ is called porous in $(P, d)$ if there exist $\alpha \in(0,1]$ and $r_{0}>0$ such that for each positive $r<r_{0}$ and each $p \in P$ there exists a ball $B(\bar{p}, \alpha r)$ of radius $\alpha r$ such that $B(\bar{p}, \alpha r) \subset B(p, r)$ and $B(\bar{p}, \alpha r) \cap \Omega$ is empty. Later on we consider a set $\Omega$ that is the complement to a set $P^{\prime}$. Then $P \backslash P^{\prime}$ is porous if there exist $\alpha>0$ and $r_{0}>0$ such that for each $r \in\left(0, r_{0}\right)$ and for each $p \in P$ an element $\bar{p}$ can be found for which $B(\bar{p}, \alpha r) \subset B(p, r) \cap P^{\prime}$.

A set $\Omega$ is called $\sigma$-porous in $(P, d)$ if $\Omega$ is a countable union of porous sets. 
Assume that the set $\mathscr{P}$ contains a strictly increasing function $l$ such that

$$
p+\lambda l \in \mathscr{P} \quad \forall p \in \mathscr{P}, \lambda>0
$$

If $\mathscr{P}$ is a convex cone, then the property (4.1) is valid. We fix such a function $l$ and assume without loss of generality that $\|l\|=1$. Note that the function $p+\lambda l$ from (4.1) is strictly increasing for each $p \in \mathscr{P}$ and each $\lambda>0$. The following definition is an extension of the definition given in [10].

Definition 4.1. A function $p \in \mathscr{P}$ is called strictly increasing with respect to $l$ if for any positive integer $n$ there exists a $\delta_{n}>0$ such that

$$
x, y \in \mathscr{B}, \quad x \leq y, \quad l(x) \leq l(y)-\frac{1}{n} \Longrightarrow p(x)<p(y)-\delta_{n}
$$

It is easy to check that each strictly increasing with respect to $l$ function is strictly increasing. Indeed, let $x<y$. Since $l$ is strictly increasing, it follows that there exists $n$ such that $l(x)<l(y)-1 / n$. Then $p(x)<p(y)-\delta_{n}$, hence $p(x)<p(y)$. Denote by $\mathscr{P}^{l}$ the set of all strictly increasing with respect to $l$ functions.

Theorem 4.2. The set $\mathscr{P} \backslash \mathscr{P}^{l}$ is $\sigma$-porous in $\left(\mathscr{P}, d_{u}\right)$.

Proof. For each positive integer $n$ consider the set $\mathscr{P}_{n}^{l}$ of all IPH functions $p \in \mathscr{P}$ such that there exists $\delta_{n}>0$ with the property (4.2). It follows from the definition of $\mathscr{P}^{l}$ that $\mathscr{P}^{l}=\bigcap_{n=1}^{\infty} \mathscr{P}_{n}^{l}$, so

$$
\mathscr{P} \backslash \mathscr{P}^{l}=\bigcup_{n=1}^{\infty}\left(\mathscr{P} \backslash \mathscr{P}_{n}^{l}\right)
$$

Thus we need to prove that the set $\mathscr{P} \backslash \mathscr{P}_{n}^{l}$ is porous in $\mathscr{P}$.

Let $n$ be a natural number. Consider a number $r \in(0,1]$ and numbers $\alpha$ and $\gamma$ such that

$$
0<\alpha<\frac{1}{2(n+1)}, \quad \gamma=\alpha(2 n+1) r
$$

We have

$$
\frac{\gamma}{n}=\alpha\left(2+\frac{1}{n}\right) r>2 \alpha r, \quad \gamma+\alpha r=2(n+1) \alpha r<r .
$$

Let $p \in \mathscr{P}$. Consider the function $\bar{p}$ defined on $C$ by

$$
\bar{p}(x)=p(x)+\gamma l(x) .
$$


Clearly $\bar{p} \in \mathscr{P}$. Since $l$ is strictly increasing, it follows that $\bar{p}$ is also strictly increasing. We now show that $\bar{p} \in \mathscr{P}_{n}^{l}$. Indeed, let $x, y \in \mathscr{B}, x \leq y$, and $l(x)<l(y)-1 / n$. Then

$$
\bar{p}(y)-\bar{p}(x)=p(y)-p(x)+\gamma(l(y)-l(x)) \geq \gamma(l(y)-l(x)) \geq \gamma \frac{1}{n} .
$$

This means that $\bar{p} \in \mathscr{P}_{n}^{l}$ with $\delta_{n}=\gamma / n$. Since $\|l\|=1$, it follows that $d(p, \bar{p})=\gamma$.

Let $q \in \mathscr{P}$ and $d(\bar{p}, q)<\alpha r$. Using (4.5) we have

$$
d(p, q) \leq d(p, \bar{p})+d(\bar{p}, q) \leq \gamma+\alpha r<r,
$$

so $B(\bar{p}, \alpha r) \subset B(p, r)$. We now show that $q \in \mathscr{P}_{n}^{l}$. Let $x, y \in \mathscr{B}, x \leq y$, and $l(x)<l(y)-$ $1 / n$. Since $d(\bar{p}, q)<\alpha r$, it follows that $q(y) \geq \bar{p}(y)-\alpha r$ and $-q(x) \geq-\bar{p}(x)-\alpha r$. Using these inequalities and (4.7) we have

$$
q(y)-q(x) \geq \bar{p}(y)-\bar{p}(x)-2 \alpha r \geq \gamma \frac{1}{n}-2 \alpha r .
$$

Let $\delta_{n}=\gamma / n-2 \alpha r$. It follows from (4.5) that $\delta_{n}>0$. Due to (4.9) we have $q \in \mathscr{P}_{n}{ }_{n}$.

We have proved that $B(\bar{p}, \alpha r) \subset \mathscr{P}_{n}^{l}$. This means that $\mathscr{P} \backslash \mathscr{P}_{n}^{l}$ is a porous set.

Remark 4.3. Porosity results for some metric spaces of increasing functions were established in [10]. The set of IPH functions is a subset of some of these metric spaces, however a porosity result for a whole space does not imply a similar result for its subspaces. Also spaces with uniform metric were not considered in [10].

We now turn to the space $\left(\mathscr{P}_{0}, d\right)$ where $\mathscr{P}_{0}$ is the set of functions from $\mathscr{P}$ with the property $\inf _{x \in \mathscr{S}} p(x)>0$ and $d$ is a metric defined by (3.10). We need the following assertion.

Lemma 4.4. Let $M$ be a positive number. Then there exist $\alpha>0$ and $r_{0}>0$ with the following property: for each $r \in\left(0, r_{0}\right)$ there exists a number $\gamma_{1}>0$ such that

$$
\begin{gathered}
\gamma_{1}+\alpha r<r, \\
e^{\alpha r}-1<\frac{1}{M}\left(e^{\gamma_{1}}-1\right) .
\end{gathered}
$$

Proof. Assume that there exist $\alpha>0$ and $r_{0}>0$ such that

$$
M\left(e^{\alpha r}-1\right)<e^{(1-\alpha) r}-1, \quad r \in\left(0, r_{0}\right) .
$$

Then for each $r \in\left(0, r_{0}\right)$ we can find $\gamma_{1} \equiv \gamma_{1}(r)>0$ such that

$$
M\left(e^{\alpha r}-1\right)<e^{\gamma_{1}}-1<e^{(1-\alpha) r}-1 .
$$

The left-hand side inequality in (4.13) is equivalent to (4.11). The right-side inequality in (4.13) is equivalent to (4.10). Thus we only need to show that there exist $\alpha>0$ and $r_{0}>0$ such that (4.12) holds.

Consider function

$$
\varphi(r)=e^{(1-\alpha) r}-M e^{\alpha r}, \quad r \geq 0
$$


We have $\varphi^{\prime}(r)=e^{(1-\alpha) r}(1-\alpha)-M e^{\alpha r} \alpha$. Choose $\alpha$ such that $(1-\alpha) / \alpha>M$. Then $\varphi^{\prime}(0)=$ $(1-\alpha)-M \alpha>0$. Therefore there exists $r_{0}>0$ such that $\varphi(r)>\varphi(0)$ for $r \in(0, r)$. Using (4.14) we have for these $r$,

$$
e^{(1-\alpha) r}-M e^{\alpha r}>1-M
$$

which is equivalent to (4.12).

Assume that the set $\mathscr{P}_{0}$ contains a strictly increasing function $l$ such that (4.1) holds. We fix this function and assume without loss of generality that $\|l\|=1$. Denote by $\mathscr{P}_{0}^{l}$ the set of all strictly increasing with respect to $l$ functions from $\mathscr{P}_{0}$ (see Definition 4.1).

Theorem 4.5. The set $\mathscr{P}_{0} \backslash \mathscr{P}_{0}^{l}$ is $\sigma$-porous in $\left(\mathscr{P}_{0}, d\right)$.

Proof. For each natural $n$ consider the set $\left(\mathscr{P}_{0}\right)_{n}^{l}$ of all functions $p \in \mathscr{P}_{0}$ such that there exists $\delta_{n}>0$ with the property (4.2). We have

$$
\mathscr{P}_{0}^{l}=\bigcap_{n=1}^{\infty}\left(\mathscr{P}_{0}\right)_{n}^{l}
$$

For each natural $m$ consider the set

$$
Q_{m}=\left\{p \in \mathscr{P}_{0}: \inf _{x \in S} p(x) \geq \frac{1}{m},\|p\| \leq m\right\} .
$$

Since $\mathscr{P}_{0}=\bigcup_{m=1}^{\infty} Q_{m}$, it follows that

$$
\mathscr{P}_{0} \backslash\left(\mathscr{P}_{0}\right)_{n}^{l}=\bigcup_{m=1}^{\infty}\left(Q_{m} \backslash\left(\mathscr{P}_{0}\right)_{n}^{l}\right) .
$$

Due to (4.16) we have

$$
\mathscr{P}_{0} \backslash \mathscr{P}_{0}^{l}=\bigcup_{n=1}^{\infty} \bigcup_{m=1}^{\infty}\left(Q_{m} \backslash\left(\mathscr{P}_{0}\right)_{n}^{l}\right)
$$

Thus, in order to obtain the result, we need to prove that the set $Q_{m, n}:=Q_{m} \backslash\left(\mathscr{P}_{0}\right)_{n}^{l}$ is porous for each of the positive integers $n$ and $m$. We fix natural $m$ and $n$. Without loss of generality assume that $m \geq 2$. Let $M=m(4 m+1) n$. Due to Lemma 4.4 , we can find numbers $\alpha>0$ and $r_{0}>0$ such that for each $r \in\left(0, r_{0}\right)$ there exists $\gamma_{1}$ for which (4.10) and (4.11) hold. We can assume without loss of generality that $r_{0} \leq 1$. We fix a number $r \in\left(0, r_{0}\right)$ and consider a number $\gamma_{1}$ corresponding to $r$. We also need the number

$$
\gamma=\frac{1}{m}\left(e^{\gamma 1}-1\right)
$$

Note that $\gamma<1$. Indeed due to (4.10), we have $\gamma_{1} \leq(1-\alpha) r<r \leq r_{0} \leq 1$. Hence

$$
\gamma=\frac{1}{m}\left(e^{\gamma_{1}}-1\right)<\frac{1}{m}(e-1)<2 \frac{1}{m} \leq 1
$$


Let $p \in Q_{m, n}$ and $\bar{p}(x)=p(x)+\gamma l(x)$ for $x \in C$. Since (4.1) holds, it follows that $\bar{p} \in \mathscr{P}_{0}$. Let $x, y \in \mathscr{B}, x \leq y$, and $l(x)<l(y)-1 / n$. Then

$$
\bar{p}(y)-\bar{p}(x) \geq \gamma(l(y)-l(x)) \geq \gamma \frac{1}{n}
$$

so $\bar{p} \in\left(\mathscr{P}_{0}\right)_{n}^{l}$. Since $\|l\|=1, \gamma \leq 1$, and $p \in Q_{m}$ it follows that

$$
\|\bar{p}\| \leq\|p\|+\gamma \leq\|p\|+1 \leq m+1 .
$$

We now estimate $d(p, \bar{p})$. Since $p(x) \leq \bar{p}(x)$ for all $x$, it follows that

$$
\rho(p, \bar{p})=\sup _{x \in C} \frac{\bar{p}(x)}{p(x)}=\sup _{x \in C} \frac{p(x)+\gamma l(x)}{p(x)}=1+\gamma \sup _{x \in C} \frac{l(x)}{p(x)}=1+\gamma \sup \left\{\frac{l(x)}{p(x)}: x \in \mathscr{S}\right\} .
$$

Since $p \in Q_{m}$, it follows that $p(x) \geq 1 / m$ for $x \in \mathscr{Y}$. Therefore we have for $x \in \mathscr{S}$

$$
\frac{l(x)}{p(x)} \leq m l(x)=\|l\| m=m .
$$

Thus $\rho(p, \bar{p}) \leq 1+\gamma m$. It follows from $(4.20)$ that $1+\gamma m=e^{\gamma_{1}}$. Then

$$
d(p, \bar{p})=\ln \rho(p, \bar{p}) \leq \ln (1+\gamma m)=\ln e^{\gamma_{1}}=\gamma_{1} .
$$

Let $q \in \mathscr{P}_{0}$ and $d(\bar{p}, q)<\alpha r$. Since $\gamma_{1}+\alpha r<r$, we have

$$
d(p, q) \leq d(p, \bar{p})+d(\bar{p}, q)<\gamma_{1}+\alpha r<r
$$

so $B(\bar{p}, \alpha r) \subset B(p, r)$. Let $s=e^{r}-1$. Due to Proposition 3.1, we have $q(x)-p(x) \leq s p(x)$ for all $x \in C$. Since $r<r_{0} \leq 1$, it follows that $s=e^{r}-1 \leq e-1<2$. Since $p \in Q_{m}$, we have

$$
q(x) \leq(1+s) p(x) \leq 3 m, \quad x \in \mathscr{B} .
$$

We check that $B(\bar{p}, \alpha r) \subset\left(\mathscr{P}_{0}\right)_{n}^{l}$. Let $q \in B(\bar{p}, \alpha r)$ and let $x, y \in \mathscr{B}$ be vectors such that $x \leq$ $y$ and $l(x)<l(y)-1 / n$. Due to Proposition 3.1 and the inequality $d(\bar{p}, q)<\alpha r$, we have

$$
\bar{p}(y)-q(y) \leq\left(e^{\alpha r}-1\right) q(y), \quad q(x)-\bar{p}(x) \leq\left(e^{\alpha r}-1\right) \bar{p}(x),
$$

hence

$$
q(y)-q(x) \geq \bar{p}(y)-\bar{p}(x)-\left(e^{\alpha r}-1\right)(\bar{p}(x)+q(y)) .
$$

Due to (4.22), (4.23), (4.28), and (4.20) we have

$$
q(y)-q(x) \geq \frac{\gamma}{n}-\left(e^{\alpha r}-1\right)(4 m+1)=\frac{\left(e^{\gamma_{1}}-1\right)}{n m}-\left(e^{\alpha r}-1\right)(4 m+1) .
$$


Applying (4.11) we conclude that

$$
\delta_{n m}:=\frac{\left(e^{\gamma_{1}}-1\right)}{n m}-\left(e^{\alpha r}-1\right)(4 m+1)>0 .
$$

It follows from $(4.31)$ that $q \in\left(\mathscr{P}_{0}\right)_{n}^{l}$. Thus $B(\bar{p}, \alpha r) \subset\left(\mathscr{P}_{0}\right)_{n}^{l}$. We have also proved that $B(\bar{p}, \alpha r) \subset B(p, r)$. This means that the set $Q_{m, n}$ is porous.

\section{IPH functions and conormal sets}

Let $X$ be a normed space with the norm $\|\cdot\|$. Let $C \subset X$ be a convex pointed cone with the nonempty interior int $C$. Assume that $X$ is equipped with the order relation $\geq$ generated by the closed convex cone $\mathrm{cl} C$ and the norm $\|\cdot\|$ is semimonotone with respect to this order. The latter means that there exists $m>0$ such that $\|x\| \geq m\|y\|$ if $x \geq y \geq 0$. Semimonotonicity of the norm is equivalent to the normality of the cone $\mathrm{cl} C$ (see, e.g., [4]). We fix a point $y \in \operatorname{int} C$. If the norm $\|\cdot\|$ is semimonotone, then (see, e.g., [4]) the norm $\|\cdot\|$ is equivalent to the following norm $\|\cdot\|_{y}$ :

$$
\|x\|_{y}=\inf \{\lambda: x \leq \lambda y,-x \leq \lambda y\}, \quad x \in X .
$$

Clearly the norm $\|\cdot\|_{y}$ is monotone, that is, $x_{1} \geq x_{2} \geq 0 \Rightarrow\left\|x_{1}\right\| \geq\left\|x_{2}\right\|$. Note that the unit ball $\left\{x:\|x\|_{y} \leq 1\right\}$ coincides with the set $\{x \in X:-y \leq x \leq y\}$.

Assume without loss of generality that $\|\cdot\|=\|\cdot\|_{y}$. It follows from this assumption that $X$ is equipped with a monotone norm. We have

$$
\mathscr{B}:=\{x \in C:\|x\| \leq 1\}=\{x \in C: x \leq y\} .
$$

If $C$ is closed, then $\mathscr{B}=\{x: 0 \leq x \leq y\}$. If $C$ is open, then $\mathscr{B}=\{x: 0 \ll x \leq y\}$ where $x \ll y$ means that $x-y \in \operatorname{int} C$. We need the following proposition.

Proposition 5.1. Let $p: C \rightarrow \mathbb{R}_{+}$be an IPH function. Then $p$ is continuous on $\operatorname{int} C$.

Proof. Let $x_{k} \rightarrow x \in \operatorname{int} C$. Since $x \in \operatorname{int} C$, it follows that there exists a number $t$ such that $t x \geq y$, where $y \in \operatorname{int} C$ is a reference point which serves for the definition of the norm $\|\cdot\|=\|\cdot\|_{y}$. Let $\varepsilon>0$ be a number such that $1-\varepsilon t>0$. Then for large enough $k$, we have

$$
x-\varepsilon t x \leq x-\varepsilon y \leq x_{k} \leq x+\varepsilon y \leq x+\varepsilon t x .
$$

Since $p$ is IPH, we have

$$
(1-\varepsilon t) p(x)=p(x-\varepsilon t x) \leq p\left(x_{k}\right) \leq p(x+\varepsilon t x)=(1+\varepsilon t) p(x) .
$$

Thus the result follows.

We consider the cone $C$ as a topological space equipped with the natural topology of a subspace. Denote the closure, interior and boundary of a set $U \subset C$ by $\mathrm{cl}_{C}$, int ${ }_{C}, \mathrm{bd}_{C}$.

A set $V \subset C$ is called upward or conormal if $(x \in V, y \in C, y \geq x) \Rightarrow y \in V$. Consider the Minkowski cogauge $\nu_{V}$ of a conormal set $V$ :

$$
\nu_{V}(x)=\sup \{\lambda>0: x \in \lambda V\}, \quad x \in C .
$$


We assume that the supremum of the empty set is equal to 0 . The Minkowski cogauge can be defined for an arbitrary coradiant set $V$. (Recall that $V$ is coradiant if $x \in V \Rightarrow \lambda x \in V$ for all $\lambda \geq 1$.) A presentation of theory of Minkowski cogauges for coradiant sets and in a finite-dimensional space can be found in [7]. Many results from [7] can be easily generalized to an arbitrary normed space. In particular, if $V$ is closed (in the topological space $C$ ), then

$$
V=\left\{x \in C: \nu_{V}(x) \geq 1\right\} .
$$

Let $V$ be a coradiant set. Then $\nu_{V}(x)=0$ if and only if ray $R_{x}:=\{v x: \nu>0\}$ does not intersect $V$ and $\nu_{V}(x)=+\infty$ if and only if $R_{x} \subset V$. Clearly $C$ is a coradiant set and $\nu_{C}(x)=+\infty$ for all $x \in C$.

Proposition 5.2. Let $V$ be a conormal set. Then $\nu_{V}(x)>0$ for each $x \in \operatorname{int} C$.

Proof. Let $u \in V$. Since $x \in \operatorname{int} C$, it follows that there exists $\lambda>0$ such that $\lambda u \leq x$. Since $V$ is conormal and $(1 / \lambda) x \geq u$, we have $(1 / \lambda) x \in V$. Thus $\nu(x) \geq \lambda>0$.

It is easy to check that the Minkowski cogauge $\nu_{V}$ of a coradiant set $V$ is a positively homogeneous of degree one function.

Proposition 5.3. A coradiant set $V$ is conormal if and only if $\nu_{V}$ is increasing.

Proof. Let $V$ be a conormal set and $x_{1} \geq x_{2}$. Then $x_{2} \in \lambda V \Rightarrow x_{1} \in \lambda V$, so $\nu_{V}\left(x_{1}\right) \geq \nu_{V}\left(x_{2}\right)$. Thus $\nu_{V}$ is increasing. Assume now that $\nu_{V}$ is increasing. Let $x_{1} \in V$ and $x_{2} \geq x_{1}$. Since $x_{1} \in V$, it follows that $\nu_{V}\left(x_{1}\right) \geq 1$. Then $\nu_{V}\left(x_{2}\right) \geq v_{V}\left(x_{2}\right) \geq 1$. This means that $x_{2} \in V$, therefore $V$ is conormal.

Corollary 5.4. A coradiant set $V$ is conormal if and only if $\nu_{V}$ is an IPH function.

Let

$$
\mathscr{V}=\{x \in C:\|x\| \geq 1\}
$$

Since $X$ is equipped with a monotone norm, it follows that the set $\mathscr{V}$ is conormal. An easy calculation shows that $\nu_{\mathscr{V}}(x)=\|x\|$ for all $x \in C$.

Let $V$ be a closed (in the topological space int $C$ ) coradiant set. Since the level sets

$$
\left\{x \in C: \nu_{V}(x) \geq r\right\}=r\left\{x \in C: \nu_{v}(x) \geq 1\right\}=r V
$$

are closed for all $r>0$ and the level set $\left\{x \in C: \nu_{V}(x) \geq 0\right\}=C$ is also closed, it follows that $\nu_{V}$ is an upper semicontinuous (in the topological space $C$ ) function. Conversely, if $\nu_{V}$ is upper semicontinuous, then $V$ is closed.

It is easy to check that

$$
\nu_{(\alpha V)}=\frac{1}{\alpha} \nu_{V}, \quad \alpha>0
$$

for a coradiant set $V$. Indeed, we have for $x \in C$,

$$
\nu_{(\alpha V)}(x)=\sup \{\lambda>0: x \in(\lambda \alpha) V\}=\sup \left\{\frac{\lambda^{\prime}}{\alpha}: x \in \lambda^{\prime} V\right\}=\frac{1}{\alpha} \nu_{V}(x) .
$$


Note that if $V$ is coradiant, then $r V \subset V$ for $r \geq 1$. If $V_{1}, V_{2}$ are coradiant, then

$$
V_{1} \subset V_{2} \Longleftrightarrow \nu_{V_{1}} \leq \nu_{V_{2}} .
$$

Indeed, it follows from the inclusion $\left\{\lambda: x / \lambda \in V_{1}\right\} \subset\left\{\lambda: x / \lambda \in V_{2}\right\}$.

Proposition 5.5. Let $V$ be a conormal set which does not contain int $C$. Then there exists $r>0$ such that $V \subset r \mathscr{V}$ where $\mathscr{V}$ is defined by (5.7).

Proof. Let $z \in \operatorname{int} C$ be a point such that $z \notin V$. Recall that the norm $\|\cdot\|=\|\cdot\|_{y}$ in the space $X$ is defined by means of the reference point $y \in \operatorname{int} C$. Since $z \in \operatorname{int}^{C} \mathscr{C}$, it follows that there exists $r>0$ such that $r y \leq z$. Since $V$ is conormal, we have $r y \notin V$. Applying (5.2) we conclude that $r \mathscr{B}:=\{x \in C:\|x\| \leq r\}=\{x \in C: x \leq r y\}$. It follows from conormality of $V$ that $r \mathscr{B} \cap V=\varnothing$, hence $V \subset\{x \in C:\|x\|>y\} \subset r \mathscr{V}$.

Proposition 5.6. Let $V$ be a conormal set which does not contain int $C$. Then the Minkowski cogauge $\nu_{V}$ is continuous at zero.

Proof. Due to Proposition 2.1, $\nu_{V}$ is continuous at zero if and only if $\left\|\nu_{V}\right\|=\sup _{x \in \mathscr{B}} \nu_{V}(x)$ $<+\infty$. Since $\mathscr{B}=\{x \in C: x \leq y\}$ and $\nu_{V}$ is an increasing function, it follows that $\left\|\nu_{V}\right\|=$ $\nu_{V}(y)$. If $\nu_{V}(y)=+\infty$, then (see Proposition 2.2) $\nu_{V}(z)=+\infty$ for all $z \in \operatorname{int} C$. This means that the ray $R_{z} \subset V$ for all $z \in$ int $C$, that is, int $C \subset V$ which contradicts our assumption. This contradiction shows that $\left\|\nu_{V}\right\|=\nu_{V}(y)<+\infty$.

It follows from this proposition that the Minkowski cogauge $\nu_{V}$ is finite if $V$ is a conormal set which does not contain int $C$.

Proposition 5.7. Let $V$ be a conormal set. Then $\inf \left\{\nu_{V}(x): x \in \mathscr{Y}\right\}>0$ if and only if there exists $\gamma>0$ such that $V \supset \gamma \mathscr{V}$. (Recall that $\mathscr{Y}=\{x \in C:\|x\|=1\}$.)

Proof. Let $r:=\inf \left\{\nu_{V}(x): x \in \mathscr{S}\right\}>0$. Then $\nu_{V}(x) \geq r\|x\|=\nu_{\left(\gamma^{\gamma} V\right)}(x)$ with $\gamma=1 / r$, hence $V \supset \gamma^{\mathscr{V}}$. Clearly $V \supset \gamma^{\mathscr{V}} \Rightarrow \inf \left\{\nu_{V}(x): x \in \mathscr{S}\right\}=1 / \gamma>0$.

Denote by $\mathcal{N}$ the totality of all closed (in the topological space $C$ ) conormal sets $V$ that do not contain int $C$. If $V \in \mathcal{N}$, then $\nu_{V}$ is a finite lower semicontinuous IPH function which is continuous at zero. On the other hand, a finite IPH lower semicontinuous and continuous at zero function $p: C \rightarrow \mathbb{R}_{+}$coincides with the Minkowski cogauge $\nu_{V}$ of the set $V=\{x: p(x) \geq 1\}$. It is easy to check that $V \in \mathcal{N}$. Let $\mathscr{P}$ be the set of all IPH lower semicontinuous and continuous at zero functions, and let $\psi: \mathcal{N} \rightarrow \mathscr{P}$ be the mapping defined by $\psi(V)=\nu_{V}$. It is well known and can be easily proved that $\psi$ is a bijection between $\mathcal{N}$ and $\mathscr{P}$ and $\psi^{-1}(p)=\{x: p(x) \geq 1\}$.

Using mapping $\psi$, we can introduce a metric $d_{u}$ on the set $\mathcal{N}$. Namely $d_{u}\left(V_{1}, V_{2}\right)=$ $d_{u}\left(\nu_{V_{1}}, \nu_{V_{2}}\right)$. Note that this metric is antihomogeneous, that is, positively homogeneous of degree -1 :

$$
d_{u}\left(\lambda V_{1}, \lambda V_{2}\right)=\lambda^{-1} d_{u}\left(V_{1}, V_{2}\right) \quad \forall \lambda>0 .
$$

Since $\left(\mathscr{P}, d_{u}\right)$ is a complete space, it also follows that $\left(\mathcal{N}, d_{u}\right)$ is a complete space.

Consider now the space $\mathcal{N}_{0}$ of all conormal closed (in $C$ ) sets such that there exists $m>0$ with the property $V \supset m \mathscr{V}$. Let $\mathscr{P}_{0}$ be the subset of $\mathscr{P}$ which consists of functions 
$p$ such that $\inf _{x \in \mathscr{S}} p(x)>0$. Then the restriction of $\psi$ to $\mathcal{N}_{0}$ is a bijection between $\mathcal{N}_{0}$ and $\mathscr{P}_{0}$. Using this restriction we can introduce a metric $d$ on the set $\mathcal{N}_{0}$, where $d\left(V_{1}, V_{2}\right)=$ $d\left(\nu_{V_{1}}, \nu_{V_{2}}\right)$ and $d\left(\nu_{V_{1}}, \nu_{V_{2}}\right)$ is defined by (3.10). Note the metric space $\left(\mathcal{N}_{0}, d\right)$ is complete.

Recall that $d\left(\nu_{V}, \nu_{U}\right)=\ln \left(\max \rho_{1}(U, V), \rho_{2}(U, V)\right)$, where

$$
\rho_{1}(U, V)=\inf \left\{\gamma: \nu_{V} \leq \gamma \nu_{U}\right\}, \quad \rho_{2}(U, V)=\inf \left\{\gamma: \nu_{U} \leq \gamma \nu_{V}\right\}
$$

Due to (5.9), (5.11) we have

$$
d(V, U)=\ln \max \left(\sigma_{1}(V, U), \sigma_{2}(V, U)\right),
$$

where

$$
\sigma_{1}(V, U)=\inf \{\gamma: \gamma V \subset U\}, \quad \sigma_{2}(V, U)=\inf \{\gamma: \gamma U \subset V\}
$$

\section{Conormal sets and decreasing functions}

Let $C$ be a convex cone in a normed space $X$ such that int $C \neq \varnothing$ and the cone $\operatorname{cl} C$ is pointed. Assume that $X$ is equipped with the order relation $\geq$ generated by $\operatorname{cl} C$ and with the norm $\|\cdot\|_{y}$ defined by (5.1) with some $y \in \operatorname{int} C$. Consider the normed space $X_{*}=$ $X \times \mathbb{R}$ with the norm $\|(x, \alpha)\|=\max (\|x\|,|\alpha|)$ for $(x, \alpha) \in X_{*}$. Let $C_{*}=C \times(0,+\infty)$. Assume that $X_{*}$ is equipped with the order relation generated by $\operatorname{cl} C_{*}$. Let $y_{*}=(y, 1)$. We have

$$
\|(x, \alpha)\|_{y_{*}}=\inf \{\lambda: x \leq \lambda y,-x \leq \lambda y, \alpha \leq \lambda,-\alpha \leq \lambda\}, \quad(x, \alpha) \in X_{*} .
$$

It easily follows from this that

$$
\|(x, \alpha)\|=\|(x, \alpha)\|_{y_{*}}, \quad(x, \alpha) \in X_{*} .
$$

Let $V \subset C_{*}$ be a closed conormal set. The function $g_{V}: C \rightarrow[0,+\infty]$ defined by

$$
g_{V}(x)=\inf \{\alpha:(x, \alpha) \in V\}, \quad x \in C
$$

is called the lower cover of $V$. (We assume that the infimum over the empty set is equal to $+\infty$.) For a function $g: C \rightarrow[0,+\infty]$, we define the domain dom $g$ and the epigraph epi $g$ by

$$
\operatorname{dom} g=\{x \in C: 0<g(x)<+\infty\}, \quad \text { epi } g=\{(x, \alpha) \in C \times(0,+\infty): \alpha \geq g(x)\},
$$

respectively.

Proposition 6.1 [8, Propositions 6.1 and 6.2]. (1) Let $V \subset C_{*}$ be a closed conormal set. Consequently, $V$ coincides with the epigraph epi $g_{V}$ of the lower cover $g_{V}$, and $g_{V}$ is an lsc (in the topological space $C$ ) and decreasing function.

(2) Let $g: C \rightarrow[0,+\infty]$ be an lsc decreasing function. Then the set $V:=$ epi $g$ is closed (in $\left.C_{*}\right)$ and conormal. 
In the rest of this section we assume that $C$ is an open cone, so $C=\operatorname{int} C$. Then also $C_{*}=\operatorname{int} C_{*}$.

Denote by $\mathscr{D}$ the set of lower semicontinuous decreasing functions $g$ defined on $C$ and such that dom $g$ is not empty. Let $\mathcal{N}_{*}$ be the totality of conormal closed (in $C_{*}$ ) subsets $V$ of $C_{*}$ such that $V \neq \mathscr{C}_{*}$. Let $\chi: \mathscr{D} \rightarrow \mathcal{N}_{*}$ be the mapping defined by

$$
\chi(g)=\text { epi } g \text {. }
$$

Then $\chi$ is a bijection between $\mathscr{D}$ and $\mathcal{N}_{*}$. Using this bijection we can define the metric $d_{u}$ on the set $\mathscr{D}$ :

$$
d_{u}(h, g):=d_{u}(\text { epi } h, \text { epi } g)
$$

We now consider the set $\mathscr{D}_{0} \subset \mathscr{D}$ that consists of functions $g \in \mathscr{D}$ such that dom $g$ is a bounded set and $\lim _{x \rightarrow 0} g(x)<+\infty$. (Note that $\lim _{x \rightarrow 0} g(x)$ exists since $g$ is a decreasing function defined on $C=\operatorname{int} C$.)

Proposition 6.2. $g \in \mathscr{D}_{0}$ if and only if there exists $m>0$ such that epi $g \supset \mathfrak{V}_{*}$, where $\mathscr{V}_{*}=\left\{(x, \lambda) \in C_{*}:\|(x, \lambda)\| \geq 1\right\}$.

Proof. Let $g \in \mathscr{D}_{0}$. Since $m_{1}:=\lim _{x \rightarrow 0} g(x)=\sup _{x \in C} g(x)<+\infty$, it follows that $g(x)<+\infty$ for all $x \in C$, hence dom $g=\{x \in C: g(x)>0\}$. Due to the definition of the norm $\|\cdot\|:=$ $\|\cdot\|_{y}$ (see (5.1)), it follows that dom $g$ is bounded if and only if there exists $m_{2}>0$ such that $x \leq m_{2} y$ for all $x \in \operatorname{dom} g$. We also have $g(x) \leq m_{1}$ for all $x \in \operatorname{dom} g$. Let $y_{*}=(y, 1)$. Since the space $X_{*}$ is equipped with the norm $\|\cdot\|_{y_{*}}$ (see (6.2)), it follows that there exists a number $m>0$ such that $x \in \operatorname{dom} g \Rightarrow\|(x, g(x))\|<m$. We now show that epi $g \supset m V_{*}$. Let $\|(x, \lambda)\| \geq m$. First assume that $x \notin \operatorname{dom} g$. Then $(x, \lambda) \in$ epi $g$ for all $\lambda>0$. It follows from this that if $(x, \lambda) \in m \mathscr{V}$, then $(x, \lambda) \in$ epig. Let $x \in \operatorname{dom} g$, then $g(x)<m$. Let $(x, \lambda) \in$ $m \mathcal{V}$. Then $\|(x, \lambda)\| \geq m$. Since $\|x\|<m$, it follows that $\lambda \geq m>g(x)$, so $(x, \lambda) \in$ epi $g$.

Assume now that epi $g \supset m V_{*}$ with some $m>0$. Then the graph $\{(x, g(x)): x \in \operatorname{dom} g\}$ of the function $g$ is placed in the set $m^{\prime} \mathscr{B}$ with some $m^{\prime}>m$. It follows from this that $g \in D_{0}$.

The restriction of the mapping $\chi$ on $\mathscr{D}_{0}$ is a bijection between $\mathscr{D}_{0}$ and the set $\mathcal{N}_{*, 0}$ of all subsets from $\mathcal{N}_{*}$ that contain $m \mathscr{V}_{*}$ with some $m$. Using this bijection we can introduce the metric $d$ on the set $\mathscr{D}_{0}$ :

$$
d(h, g)=d(\text { epi } h, \text { epi } g)
$$

where $d$ (epi h, epig) is defined by (5.14). Due to (5.14) and (5.15), we have

$$
d(h, g)=\ln \max \left(\sigma_{1}(\text { epi } h, \text { epi } g), \sigma_{2}(\text { epi } h, \text { epi } g)\right),
$$

where

$$
\begin{aligned}
& \sigma_{1}(\text { epi } h, \text { epi } g)=\inf \{\gamma: \gamma \text { epi } h \subset \text { epi } g\}, \\
& \sigma_{2}(\text { epi } h, \text { epi } g)=\inf \{\gamma: \gamma \text { epi } g \subset \text { epi } h\} .
\end{aligned}
$$

We need the following assertion. 
Lemma 6.3. Let $g, h \in \mathscr{D}_{0}$ and $\gamma>0$. Then $\gamma$ epi $g \subset$ epi $h$ if and only if

$$
\gamma g(x) \geq h(\gamma x), \quad x \in C .
$$

Proof. Let $\gamma$ epi $g \subset$ epi $h$ and $x \in C$. Then $(\gamma x, \gamma g(x)) \in$ epi $h$, therefore $\gamma g(x) \geq h(\gamma x)$. Assume now that $(6.10)$ holds and $(x, v) \in$ epig. Then

$$
v \geq g(x) \geq \frac{1}{\gamma} h(\gamma x)
$$

that is, $\gamma \nu \geq h(\gamma x)$. This means that $\gamma(x, \nu) \in$ epi $h$.

It follows from this lemma that

$$
\begin{aligned}
& \xi_{1}(h, g):=\sigma_{1}(\text { epi } h, \text { epi } g)=\inf \left\{\gamma: g(x) \geq \frac{1}{\gamma} h(\gamma x), x \in C\right\}, \\
& \xi_{2}(h, g):=\sigma_{2}(\text { epi } h, \text { epi } g)=\inf \left\{\gamma: h(x) \geq \frac{1}{\gamma} g(\gamma x), x \in \operatorname{dom} h\right\} .
\end{aligned}
$$

It follows from the aforesaid that

$$
d(h, g)=\max \left(\xi_{1}(h, g), \xi_{2}(h, g)\right), \quad h, g \in \mathscr{D}_{0},
$$

where $\xi_{1}(h, g), \xi_{2}(h, g)$ are defined by $(6.12)$ and (6.13), respectively.

\section{Strictly conormal sets and strictly decreasing functions}

Let $C$ be an open convex cone in a Banach space $X$. Assume that the cone $\mathrm{cl} C$ is pointed and $X$ is equipped with the order relation $\geq$ generated by $\mathrm{cl} C$. Assume that the norm in $X$ coincides with the norm $\|\cdot\|_{y}$ defined by (5.1) with $y \in C$. Let $\mathcal{N}$ be the set of all conormal closed (in $C$ ) subsets of $C$ that are different from $C$. It is easy to see that for each $V \in \mathcal{N}$, the boundary $\operatorname{bd}_{C} V$ is nonempty. Indeed, as it is shown in the proof of Proposition 7.1 below, the set $\left\{x: \nu_{V}(x)=1\right\}$ coincides with $\operatorname{bd}_{C} V$.

A conormal set $U \subset \mathcal{N}$ is called strictly conormal if for each boundary point $x \in \operatorname{bd}_{C} U$, the inequality $z<x \Rightarrow z \notin U$. (By definition, $z<x \Leftrightarrow z \leq x$ and $z \neq x$.)

Proposition 7.1. Let $V \in \mathcal{N}$. Then the Minkowski cogauge $\nu_{V}$ is strictly increasing if and only if $V$ is strictly conormal.

Proof. (1) Let $\nu_{V}$ be strictly increasing. Due to Proposition 5.1, $v_{V}$ is continuous on the open cone $C$. We also have $V=\left\{x \in C: \nu_{V}(x) \geq 1\right\}$. It easily follows from this that $\operatorname{bd}_{C} V=\left\{x: \nu_{V}(x)=1\right\}$. Let $x \in \operatorname{bd}_{C} V$. If $z<x$, then $\nu_{V}(z)<\nu_{V}(x)=1$, hence $z \notin V$. We proved that $V$ is strictly normal.

(2) Let $V$ be a strictly conormal set and let $x>z$. Since $0<\lambda:=\nu_{V}(x)<+\infty$, we can consider the elements $x^{\prime}=x / \lambda$ and $z^{\prime}=z / \lambda$. Clearly $x^{\prime}>z^{\prime}$. Since $\nu_{V}\left(x^{\prime}\right)=1$, it follows that $x^{\prime} \in \operatorname{bd}_{C} V$, therefore $z^{\prime} \notin V$. This means that $\nu_{V}\left(z^{\prime}\right)<1$. Due to positive homogeneity of $\nu_{V}$, we have $\nu_{V}(z)<\nu_{V}(x)$.

Consider a decreasing function $g \in \mathscr{D}$ defined on $C$. Let $V=\operatorname{epi} g$. Then $V \subset C_{*}:=$ $C \times(0,+\infty)$. 
Proposition 7.2 [8, Theorem 7.1]. Let $g \in \mathscr{D}$. The following statements are equivalent:

(i) the set epig is strictly conormal;

(ii) the function $g$ is strictly decreasing on the set dom $g$ and continuous (the latter means that $g\left(x_{n}\right) \rightarrow g(x)$ if $x_{n} \rightarrow x$ for all $x \in \operatorname{int} C$, including $\left.x \notin \operatorname{dom} f\right)$.

Using results of Section 4, we can show that the complements to the totality of strictly conormal sets and the totality of strictly decreasing and continuous functions are $\sigma$ porous in some metrics. Let $C$ be an open convex cone in Banach space $X$ with a semimonotone norm. Consider sets $\mathcal{N}, \mathcal{N}_{0}$ and $\mathscr{D}, \mathscr{D}_{0}$. The following results follow from Theorem 4.2 and the definition of the metric $d_{u}$ in $\mathcal{N}$ and $\mathscr{D}$.

THeOREM 7.3. Assume that the set $\mathcal{N}$ contains a strictly conormal set. Then the complement to the totality of all strictly conormal sets $U \subset \mathcal{N}$ is $\sigma$-porous in the metric space $\left(\mathcal{N}, d_{u}\right)$.

Theorem 7.4. Assume that the set $\mathscr{D}$ contains a function $g^{\prime}$ that is strictly decreasing on dom $g^{\prime}$ and continuous. Then the complement to the totality of all functions $g \in \mathscr{D}$, that are strictly decreasing on dom $g$ and continuous, is $\sigma$-porous in the metric space (D, $\left.d_{u}\right)$.

The following results follow from Theorem 4.5 and the definition of the metric $d$ in $\mathcal{N}_{0}$ and $\mathscr{D}_{0}$.

TheOREm 7.5. Assume that the set $\mathcal{N}_{0}$ contains a strictly conormal set. Then the complement to the totality of all strictly conormal sets $V \in \mathcal{N}_{0}$ is $\sigma$-porous in the metric space $\left(\mathcal{N}_{0}, d\right)$.

Theorem 7.6. Assume that the set $\mathscr{D}_{0}$ contains a function $g^{\prime}$ that is strictly decreasing on $\operatorname{dom} g^{\prime}$ and continuous. Then the complement to the totality of all functions $g \in \mathscr{D}_{0}$, that are strictly decreasing on dom $g$ and continuous, is $\sigma$-porous in the metric space $\left(\mathscr{D}_{0}, d\right)$.

\section{Application to optimization}

We now give some application of the results obtained to optimization. Let $f$ and $g$ be real-valued functions defined on the $n$-dimensional space $\mathbb{R}^{n}$. Consider the following optimization problem $P(f, g)$ :

$$
\text { minimize } f(x) \text { subject to } g(x) \leq 0, \quad i=1, \ldots, m \text {. }
$$

For each $y \in \mathbb{R}$ consider the set $A(y)=\{x \in X: g(x) \leq y\}$. We assume that the set $A(0)$ of feasible elements is nonempty, then $A(y)$ is nonempty for all $y \geq 0$. The function $\beta_{f, g}$ defined on $\mathbb{R}_{+}$by

$$
\beta_{f, g}(y)=\min \{f(x): x \in A(y)\}, \quad y \geq 0
$$

is called the perturbation function of the problem $P(f, g)$. It follows directly from the definition that $\beta_{f, g}$ is a decreasing function and $\beta_{f, g}(0)$ coincides with the optimal value $\inf \{f(x): g(x) \leq 0\}$ of $P(f, g)$.

Remark 8.1. Note that $\beta_{f, g}(0) \geq \beta_{f, g}(y)$ for $y>0$, so $\beta_{f, g}$ is upper semicontinuous at the origin. Below we impose assumptions that guarantee lower semicontinuity of $\beta_{f, g}$. If these assumptions hold, then $\beta_{f, g}$ is continuous at zero. Sometimes we can consider $\beta_{f, g}$ 
as a function defined on $(0,+\infty)$. It follows from the aforesaid that this will not lead to a misunderstanding.

We will use the following assumptions:

(1) $f$ and $g$ are continuous functions;

(2) $f$ is nonnegative and $0=\min _{x \in \mathbb{R}^{n}} f(x)<\min _{x \in A(0)} f(x)$;

(3) $A(y)=\operatorname{cl}\{x: g(x)<y\}$ for all $y \geq 0$;

(4) the sets $A(y)$ are compact for $y \geq 0$.

We discuss assumption (2). The inequality $\min _{x \in \mathbb{R}^{n}} f(x)<\min _{x \in A(0)} f(x)$ means that the constraint $g(x) \leq 0$ is essential: the constrained minimum of $f$ is greater than its unconstrained minimum. Let $\tilde{f}: \mathbb{R}^{n} \rightarrow \mathbb{R}$ be an arbitrary continuous function, which attends its global minimum on $\mathbb{R}^{n}$ and such that $\min _{x \in \mathbb{R}^{n}} \tilde{f}(x)<\min _{x \in A(0)} \tilde{f}(x)$. Let $f(z)=$ $\tilde{f}(z)-\min _{x \in \mathbb{R}^{n}} \tilde{f}(x)$. Then assumption (2) holds for the function $f$. Since the optimization problem $P(\tilde{f}, g)$ is equivalent to $P(f, g)$, we can conclude that assumption (2) is not very restrictive.

Assumptions (3) and (4) describe some properties of the constraint $g$. If $g(0)<0$, then the interior of the set int $A(0)$ of feasible elements is not empty. The equality $A(y)=\operatorname{cl}\{x$ : $g(x)<y\}$ can be considered as a certain constraint qualification. If $g$ is coercive, that is, $\lim _{\|x\| \rightarrow+\infty} g(x)=+\infty$, then assumption (4) holds.

We consider $\mathbb{R}$ as a Banach space with the norm $\|x\|=|x|$ and with the open cone $C=$ $(0,+\infty) \subset \mathbb{R}$. Recall that the set $\mathscr{D}_{0}$ consists of all decreasing finite lower semicontinuous functions defined on $C$ such that the set dom $g$ is bounded.

Proposition 8.2. The perturbation function $\beta_{f, g}$ belongs to $\mathscr{D}_{0}$.

Proof. The decreasing function $\beta_{f, g}$ is finite since $\beta_{f, g}(0)<+\infty$. Lower semicontinuity of $\beta_{f, g}$ follows from compactness of the set $A(y)$. (See, e.g., [9, Proposition 3.15], where the lower semicontinuity of $\beta_{f, g}$ at zero is proved. The same proof can also be used for points $y \neq 0$.) Let $z$ be a global minimum of $f$ and let $y>0$ be a number such that $z \in A(y)$. Then $\beta_{f, g}\left(y^{\prime}\right)=0$ for $y^{\prime}>y$, hence $\operatorname{dom} \beta_{f, g}$ is bounded.

Proposition 8.3. Let $\beta \in \mathscr{D}_{0}$. Then there exists a problem $P(f, g)$ such that assumptions (1)-(4) hold and the perturbation function $\beta_{f, g}$ of $P(f, g)$ coincides with $\beta$.

Proof. Let $g$ be a continuous function defined on $\mathbb{R}^{n}$ and mapping onto $\mathbb{R}$ such that assumptions (3) and (4) hold. Let $f(x)=\beta\left(g^{+}(x)\right)$ where $g^{+}(x)=\max (g(x), 0)$. Then $f$ is lower semicontinuous and nonnegative. We also have $f(x)=0$ for all $x$ such that $g(x) \notin \operatorname{dom} \beta$. Thus $\min _{x \in \mathbb{R}^{n}} f(x)=0$. Let $y>0$. Since $g$ maps onto $\mathbb{R}$, it follows that there exists $z \in \mathbb{R}^{n}$ such that $g(z)=y$. Since $\beta$ is decreasing, we have

$$
\beta_{f, g}(y)=\inf \{f(x): g(x) \leq y\}=\inf \left\{\beta\left(g^{+}(x)\right): g(x) \leq y\right\}=\beta(g(z))=\beta(y) .
$$

The following assertion shows that if $P(f, g)$ has no global minimizers on the boundary of the set of feasible elements, then the perturbation function $\beta_{f, g}$ is not strictly decreasing on the set $\operatorname{dom} \beta_{f, g}$. 
Proposition 8.4 [9, Proposition 3.16]. Assume that

$$
M:=\min \{f(x): g(x) \leq 0\}<\min \{f(x): g(x)=0\} .
$$

Then there exists $\eta>0$ such that the perturbation function $\beta_{f, g}$ is constant (and equal to $\left.\beta_{f, g}(0) \equiv M\right)$ on the segment $[0, \eta)$.

CoRollary 8.5. If $\beta_{f, g}$ is a strictly decreasing function in a neighborhood of zero, then

$$
\min \{f(x): g(x) \leq 0\}=\min \{f(x): g(x)=0\},
$$

that is, a global minimizer of $P(f, g)$ is located on the boundary of the set of feasible elements. The converse statement does not hold. (Consider, e.g., $f(x)=c$ for all $x$ and an arbitrary function $g$.)

The theoretical study of the optimization problem $P(f, g)$ can be accomplished with the help of the perturbation function $\beta_{f, g}$. We can identify two optimization problems if they possesses the same perturbation function. Consider the set $H$ of all pairs $(f, g)$, where $f, g$ are functions defined on $\mathbb{R}^{n}$ and mapping into $\mathbb{R}$, such that assumptions (1)(4) hold. We say that pairs $(f, g) \in H$ and $\left(f^{\prime}, g^{\prime}\right) \in H$ are equivalent if $\beta_{f, g}=\beta_{f^{\prime}, g^{\prime}}$. Denote by $\mathscr{H}$ the set of all classes of equivalent pairs. For each $\pi \in \mathscr{H}$, denote by $\beta_{\pi}$ the function $\beta_{f, g}$ with $(f, g) \in \pi$. We can identify an element $\pi \in \mathcal{H}$ with the function $\beta_{\pi}$. Hence we can identify $\mathscr{H}$ with $\mathscr{D}_{0}$. Let

$$
d\left(\pi_{1}, \pi_{2}\right)=d\left(\beta_{\pi_{1}}, \beta_{\pi_{2}}\right), \quad \pi_{1}, \pi_{2} \in \mathscr{H},
$$

where $d\left(\beta_{\pi_{1}}, \beta_{\pi_{2}}\right)$ is defined by (6.8). Then the metric space $(\mathscr{H}, d)$ is isometric to $\left(\mathscr{D}_{0}, d\right)$. It is clear that $(\mathcal{H}, d)$ is a complete space.

Let $\mathcal{H}^{\prime}$ be the set of classes $\pi$ of equivalent pairs $(f, g)$ such that $\pi$ contains at least one pair $(f, g)$ for which $\min \{f(x): g(x) \leq 0\}<\min \{f(x): g(x)=0\}$ (i.e., the optimization problem $P(f, g)$ has no solutions on the boundary of the set of feasible elements). Then the function $\beta_{\pi}$ is not strictly increasing on $\operatorname{dom} \beta_{\pi}$. It follows from Theorem 7.6 that the following result holds.

Theorem 8.6. The set $\mathscr{H}^{\prime}$ is $\sigma$-porous in $(\mathscr{H}, d)$.

Remark 8.7. $\sigma$-porosity for optimization problems was studied by Zaslavski [11]. Consider a nonempty closed subset $Z$ of a complete metric space $X$ such that $Z=\operatorname{clint} Z$. Consider also a complete metric space $M$ of continuous functions $f: X \rightarrow \mathbb{R}$ that are bounded from below on $Z$ with the metric $d(f, g)=r(f, g)(1+r(f, g))^{-1}$ where $r(f, g)=$ $\sup \{|f(x)-g(x)|: x \in X\}$. The following result holds [11].

There exists a set $\mathscr{F} \subset \mathcal{M}$ which is a countable intersection of open everywhere dense subsets such that for each $f \in \mathcal{M}$ the minimization problem, minimize $f(x)$ subject to $x \in C$ has a unique solution which is an interior point of $C$.

At first glance this result contradicts Theorem 8.6. However, there is no contradiction here. Indeed, metric spaces $\mathscr{H}$ and $\mathcal{M}$ are very different. The first of them contains equivalent pairs of optimization problems that satisfy assumptions (1)-(4) and the second contains only objective functions with the fixed set $C$ of feasible elements. Metrics in 
$\mathscr{H}$ and $\mathcal{M}$ are also very different. Thus we have a new confirmation of the well-known fact that generic properties strongly depend on the metric space under consideration.

\section{References}

[1] F. S. De Blasi and J. Myjak, On a generalized best approximation problem, J. Approx. Theory 94 (1998), no. 1, 54-72.

[2] R. Deville and J. P. Revalski, Porosity of ill-posed problems, Proc. Amer. Math. Soc. 128 (2000), no. $4,1117-1124$.

[3] J. Dutta, J. E. Martínez-Legaz, and A. M. Rubinov, Monotonic analysis over cones. I, Optimization 53 (2004), no. 2, 129-146.

[4] M. A. Krasnosel'skiı̌, Positive Solutions of Operator Equations, (L. F. Boron, ed.), P. Noordhoff, Groningen, 1964, translated from Russian by R. E. Flaherty.

[5] S. Reich and A. J. Zaslavski, Asymptotic behavior of dynamical systems with a convex Lyapunov function, J. Nonlinear Convex Anal. 1 (2000), no. 1, 107-113.

[6] - The set of divergent descent methods in a Banach space is $\sigma$-porous, SIAM J. Optim. 11 (2001), no. 4, 1003-1018.

[7] A. M. Rubinov, Abstract Convexity and Global Optimization, Nonconvex Optimization and Its Applications, vol. 44, Kluwer Academic Publishers, Dordrecht, 2000.

[8] Monotonic analysis: convergence of sequences of monotone functions, Optimization 52 (2003), no. 6, 673-692.

[9] A. M. Rubinov and X. Yang, Lagrange-Type Functions in Constrained Non-Convex Optimization, Applied Optimization, vol. 85, Kluwer Academic Publishers, Massachusetts, 2003.

[10] A. M. Rubinov and A. J. Zaslavski, Two porosity results in monotonic analysis, Numer. Funct. Anal. Optim. 23 (2002), no. 5-6, 651-668.

[11] A. J. Zaslavski, On a generic existence result for a class of optimization problems, preprint, 2003.

A. M. Rubinov: School of Information Technology and Mathematical Sciences (SITMS), Centre for Informatics and Applied Optimization, University of Ballarat, Ballarat Victoria 3353, Australia

E-mail address: a.rubinov@ballarat.edu.au 


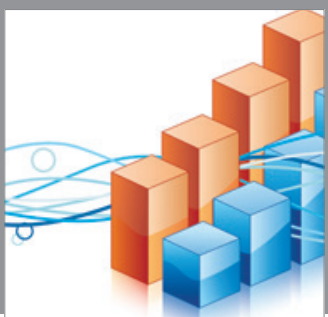

Advances in

Operations Research

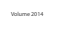

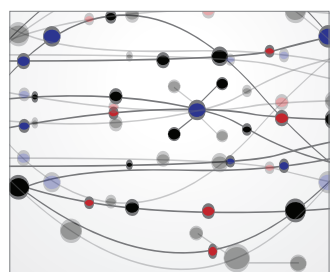

\section{The Scientific} World Journal
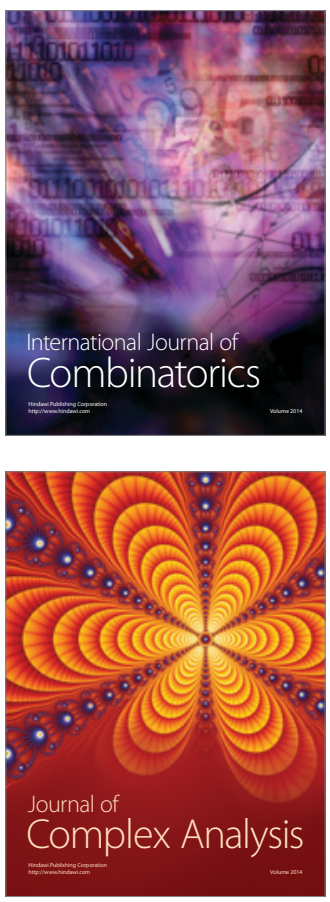

International Journal of

Mathematics and

Mathematical

Sciences
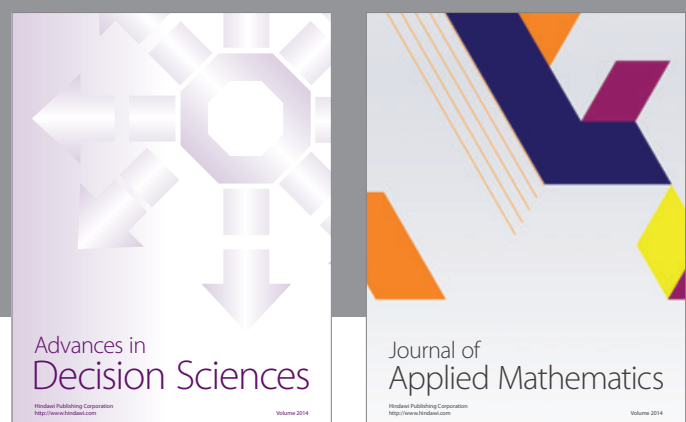

Journal of

Applied Mathematics
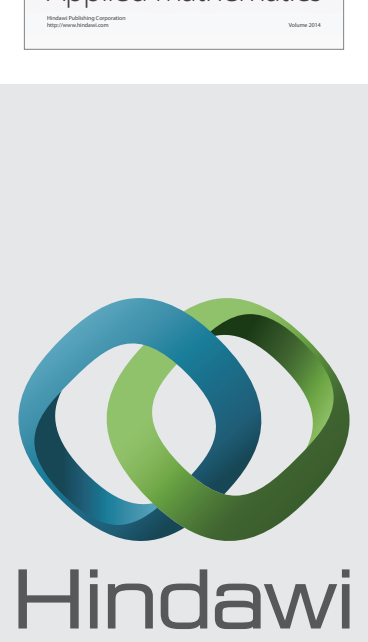

Submit your manuscripts at http://www.hindawi.com
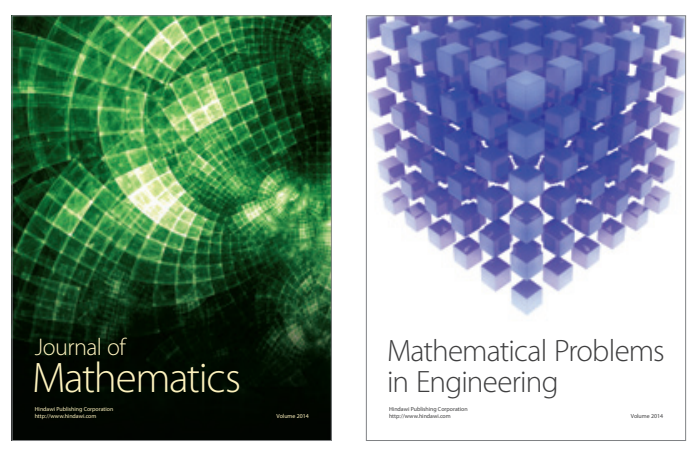

Mathematical Problems in Engineering
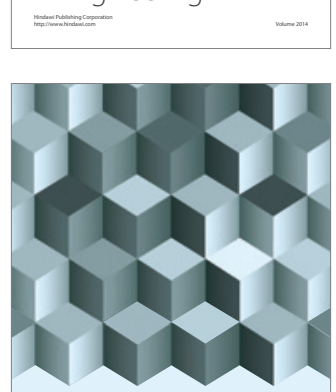

Journal of

Function Spaces
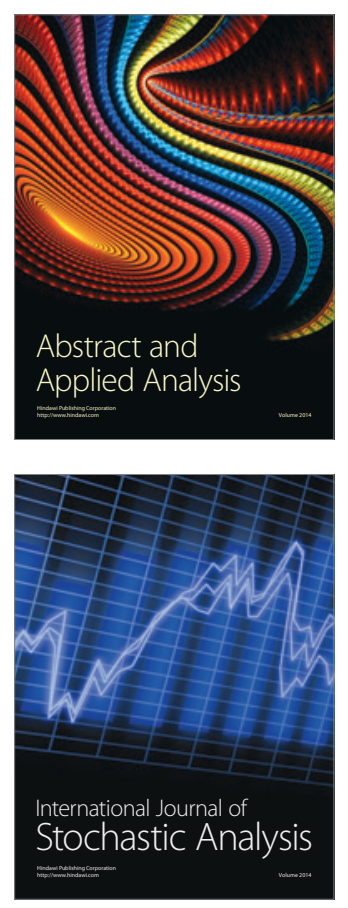

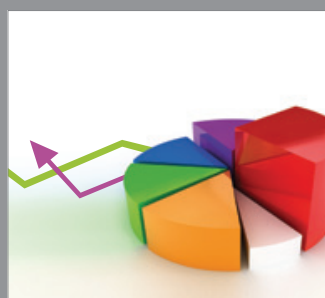

ournal of

Probability and Statistics

Promensencen
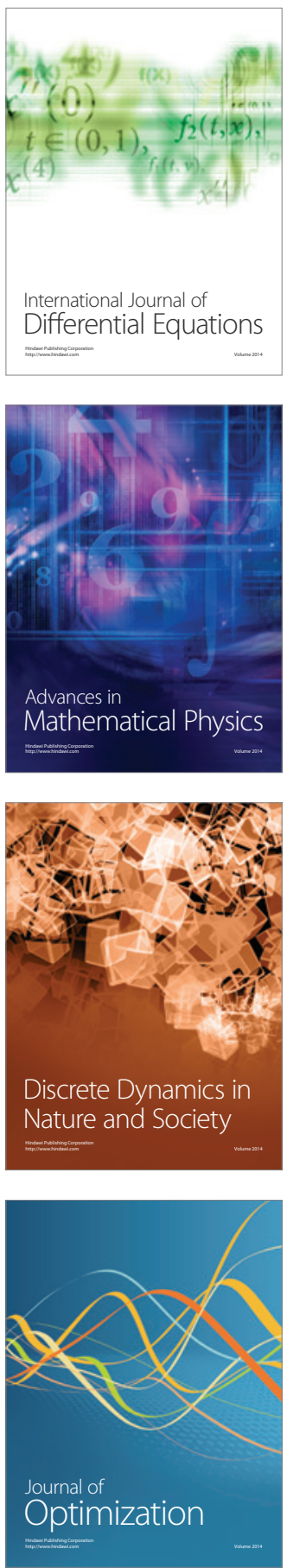